\title{
Inherited bone marrow failure syndromes: an overview
}

\author{
Shovana Karki \\ Department of Pathology, Maharajgunj Medical Campus, TUTH, Kathmandu, Nepal.
}

\section{Keywords: \\ Cytopenia; \\ Diamond-Blackfan \\ anemia; \\ Dyskeratosis congenita; \\ Fanconi anemia; \\ Inherited bone marrow \\ failure;}

\begin{abstract}
Inherited bone marrow failure syndromes are a diverse set of genetic disorders characterized by insufficient blood cell production leading to cytopenias/pancytopenia. Bone marrow failure can be restricted to one or two blood cell lineages, with symptoms specific to the particular cell lineage or it can affect all cell lineages, causing clinical symptoms similar to aplastic anemia. Inherited bone marrow failure syndromes are genetically heterogeneous diseases resulting from germline mutations that affect key cellular pathways namely ribosomal biogenesis, telomerase biology, DNA repair, and structural proteins. Common Inherited bone marrow failure syndromes are Diamond-Blackfan anemia, Fanconi anemia, Dyskeratosis Congenita, and Shwachman-Diamond syndrome. These different syndromes have variable prognoses and risks of developing hematological or solid malignancies. Thus the accurate diagnosis of these diseases differentiating it from other Inherited bone marrow failure syndromes and other causes of bone marrow failure is of utmost importance for management and surveillance of long-term squeal of the disease. Other causes of BMF can be acquired. The most common forms of Bone marrow failure can occur from chemicals, radiation, drugs, viral infections, immune disorders, myelodysplastic syndrome, paroxysmal nocturnal hemoglobinuria, or large granular lymphocytic leukemia. Moreover, these Inherited bone marrow failure syndromes are often heritable, affecting other family members, thus requiring insightful genetic counseling. This review discusses the frequent Inherited bone marrow failure syndromes along with their differential diagnosis.
\end{abstract}

\section{Correspondence:}

Dr. Shovana Karki, $M D$

Associate Professor: Department of Pathology,

Maharajgunj Medical Campus, TUTH, Maharajgunj, Kathmandu, Nepal

ORCID ID: 0000-0001-5429-2422

Email: shovana_karki@hotmail.com

Received : September $30^{\text {th }} 2020$; Accepted : December $28^{\text {th }} 2020$

Citation: Karki S. Inherited bone marrow failure syndromes: an overview. J Pathol Nep 2021;11(1): 1873-80.DOI: $10.3126 /$ jpn.v11i1.31635

Copyright: This is an open-access article distributed under the terms of the Creative Commons Attribution 4.0 International License, which permits unrestricted use, distribution, and reproduction in any medium, provided the original author and source are credited.

\section{INTRODUCTION}

Bone marrow failure $(\mathrm{BMF})$, a rare disease, is a lifethreatening condition caused by ineffective/defective hematopoiesis in the marrow resulting in cytopenia/ pancytopenia in the peripheral blood and diminished production of one or more hematopoietic cell lineages in the bone marrow. ${ }^{1,2}$ They can be categorized as 1) Inherited bone marrow failure (IBMF) and 2) Acquired bone marrow failure.

Acquired or inherited BMF can be seen in both adults and children. But IBMF syndromes are more frequently seen in children. Inherited bone marrow failure syndromes are genetic diseases characterized by bone marrow failure, 
physical abnormalities, and a genetic predisposition to developing malignancies. The most common IBMF syndromes are: 1) Diamond-Blackfan anemia (DBA), 2) Fanconi anemia (FA), 3) Dyskeratosis congenita (DC), and 4) Shwachman-Diamond syndrome (SDS). These syndromes show overlaps in their clinical and hematological manifestation. However, different syndromes have variable prognoses and risks of developing hematological or solid malignancies. ${ }^{3}$ Thus accurate diagnosis of the disease is of utmost importance for management and surveillance of long-term squeal of the disease. ${ }^{4}$ This review discusses the frequent IBMS syndromes along with their differential diagnosis.

IBMF syndromes are genetically heterogeneous diseases resulting from germline mutations that affect key cellular pathways namely ribosomal biogenesis, telomerase biology, DNA repair, and structural proteins. ${ }^{3}$ In these IBMF syndromes, there is no genotypic-phenotypic correlation eg. mutation affecting the same cellular pathway like ribosomal biosynthesis- can lead to different phenotypes- as a pure red cell aplasia in DBA, neutropenia in SDS, or progressive marrow failure in DC. ${ }^{3}$ Besides, the same phenotype i.e anemia with reticulocytopenia can be due to mutations affecting the protein responsible for ribosomal synthesis as in DBA or mutations in structural proteins as in CDA. ${ }^{3}$

\section{BONE MARROW FAILURE SYNDROMES ASSOCIATED WITH PURE RED CELL APLASIA}

\section{Diamond-Blackfan Anemia}

Diamond-Blackfan Anemia (DBA) is the most frequent cause of isolated red cell production failure. The majority present in the first year of life as transfusion-dependent anemia. But in non-classical DBA, they can present later in life. $^{3}$ Classical DBA affects seven per million live births. ${ }^{5}$ Congenital abnormalities including dysmorphic faces, short stature, eye, kidney, and hand abnormalities can be seen in approximately half of the patients with DBA. ${ }^{5}$ Malignancies associates with this inherited syndrome are acute myeloid leukemia (AML), myelodysplastic syndrome (MDS), colon cancers, female genital cancers, and osteosarcomas. ${ }^{6}$ The cumulative incidence of developing solid tumors by the age of 70 years is $50 \%$ whereas hematological malignancy like MDS and AML is $5 \%$ by the age of 50 and $5 \%$ by the age of 46 , respectively. ${ }^{6,7}$ The median age at diagnosis is about three months. They typically present with macrocytic anemia with reticulocytopenia. Elevated adenosine deaminase (eADA) activity and $\mathrm{HbF}$, strong expression of antigen on red blood cells, elevated vitamin B12, and folate is also seen. The median age for survival is 67 years. ${ }^{7}$ Treatment-related cause, infections, and complications of iron overload or hematopoietic stem cell transplant (HSCT) are the major causes of death in DBA. ${ }^{4}$

Unlike other IBMF syndromes, in DBA, bone marrow is usually normocellular for age. Regardless of the normal cellularity of the marrow, erythroid hypoplasia is noted. Erythroid precursors are eitherabsent or decreased. ${ }^{5}$ Erythroid precursors are sparsely distributed in the marrow, however, in some cases, a few small clusters, almost always consisting of pro-erythroblasts can be seen. Immunohistochemical (IHC) marker CD71 is useful in identifying these precursors and their distribution. ${ }^{8}$ Megaloblastic changes, mimicking enlarged pro-erythroblasts seen in Parvovirus B19 infection may be displayed in the erythroid series. ${ }^{2}$ Mild dysplastic changes in granulopoiesis and erythropoiesis may be present. Lymphocytes are elevated which may be physiological in infancy. ${ }^{9}$ Hematogones are also elevated. CD 34 and TdT positive immature cells may be increased. ${ }^{10} \mathrm{p} 53$ staining in the erythroid precursors from a patient with DBA in bone marrow biopsy has been reported. The variable staining pattern is observed depending on p53 mutation and disease status. ${ }^{11}$

The main differential diagnosis to be considered in older children include transient erythroblastopenia of childhood (TEC), acquired causes of red cell aplasia like Parvovirus B19 infection, HIV or other infections, drug-induced or immune-mediated red cell aplasia. There will be no significant history of anemia prior to the onset of the disease in acquired causes. ${ }^{3}$ TEC is typically seen between 3 months and 4 years of age ${ }^{12}$ and usually persists for almost a month. ${ }^{13}$ Though its etiology is unknown, a transient autoimmune mechanism that is triggered by infection or an unknown environmental factor in a genetically susceptible host is suspected. ${ }^{12-14}$ Diagnostic challenge between TEC and DBA is due to their overlapping morphological features. Both disorders present with erythroid hypoplasia, absent or reduced erythroid precursors, and normal granulopoiesis and megakaryopoiesis in bone marrow (BM). However, it is noted that the BM of DBA patients frequently has higher cellularity than TEC patients, possibly because bone marrows are evaluated at an earlier age for DBA. TEC patients can have pronounced lymphocytosis ${ }^{15}$ or erythroid hyperplasia ${ }^{16}$ in the recovery phase in the bone marrow, which is less pronounced in DBA.

The diagnosis of MDS/AML secondary to DBA should be made carefully, as this entity has a low incidence and occurs late in life. ${ }^{7,6}$ In the neonates, when BM biopsies are frequently performed for the DBA diagnosis, histomorphological features mimicking clonal evolution are exhibited: BM hypercellularity, increased blast cells, and mild dyshematopoiesis. Histomorphological evaluations along with comparisons with the baseline bone marrow and concurrent chromosomal studies are required for an accurate diagnosis. ${ }^{5}$ The demonstration of known chromosomal abnormalities associated with sporadic MDS/ AML or multiple chromosomal abnormalities is considered a warning finding. ${ }^{17,18}$

In the first year of life, the differential diagnosis of DBA includes congenital dyserythropoietic anemia (CDA), Pearson syndrome, Hemoglobinopathies, Hereditary 
hemolytic anemias, and immune-mediated hemolytic anemia. In Pearson syndrome, in the bone marrow vacuolations of the precursor cells and increased ringed sideroblasts are found which is not associated with DBA. Increased hemoglobin degradation products with splenomegaly are found in hemolytic anemia which are not usual to DBA. ${ }^{3}$

DBA is a genetic disease with a mixed pattern including $\mathrm{AR}, \mathrm{AD}$, and possibly X-linked inheritance. ${ }^{19}$ Anemia in DBA is usually severe with a need for transfusion. They are treated with steroids. For refractory cases, allogeneic hematopoietic stem cell transplant is the option.

\section{BONE MARROW FAILURE SYNDROMES ASSOCIATED WITH MULTIPLE CYTOPENIAS}

\section{Fanconi Anemia}

Fanconi anemia (FA) is the commonest IBMF syndrome characterized by progressive bone marrow failure, congenital abnormalities, chromosomal fragility, and cancer predisposition. ${ }^{1,20}$ Commonly encountered congenital abnormalities are short stature, skin lesions as café au lait spots or hypo- and hyper-pigmentation, and abnormalities of the upper limbs. In $20-25 \%$ of the cases, microcephaly, microphthalmia, hypogonadism, and structural renal abnormalities present. At least one abnormality is reported in $60 \%$ of cases. Diagnosis can be delayed due to a lack of abnormalities in these cases.

FA typically presents as bone marrow failure between 5-15years. ${ }^{20}$ The mean age at presentation is 6.5years. 1 Pancytopenia is the commonest manifestation of the disease, though macrocytosis and persistence of $\mathrm{Hb} \mathrm{F}$ are also observed. Thrombocytopenia usually precedes neutropenia and anemia. By 40 years of age, the cumulative frequency of BMF is $90 \%{ }^{21}$ Patients with birth defects are likely to develop BMF while the absence of these defects are associated with a higher risk of developing acute leukemia and solid tumors in adulthood. The relative risk of developing head and neck squamous cell carcinoma (SCC) is 500-fold and AML is 600-fold higher in FA as opposed to the general population. ${ }^{3}$ By 65 years, in patients with FA, the cumulative frequency for solid tumors is about $20 \%$ and for MDS and leukemia is $50 \%$ by 50 years and $5 \%$ by 30 years, respectively. ${ }^{7}$ The median age for survival is 29 years and complications from BMF, HSCT and malignancies are major causes of death.

In FA, BM is usually hypocellular for age. ${ }^{1,22}$ Variable degree of cellularity is present depending on the time of the biopsy and the stage of the disease. In a study, $\mathrm{BM}$ examination at the first detection of a hematologic abnormality found reduced cellularity in $75 \%$ of cases, normal or increased cellularity in $13 \%$, and MDS or AML in $12 \%$ of cases. ${ }^{23} \mathrm{BM}$ may be normocellular in an early stage of the disease, however, it may become aplastic or hypocellular with increasing severity of BMF.2 By 10 years of age, approximately $80 \%$ of patients develop BMF. ${ }^{21} \mathrm{BM}$ may exhibit severe hypocellularity or fatty marrow with loss of myeloid and erythroid precursors and megakaryocytes in the aplastic phase. These features are histologically indistinguishable from other forms of aplastic marrow, such as idiopathic or acquired aplastic anemia or other IBMF syndromes. ${ }^{2}$ A comprehensive clinical assessment and, if needed, a chromosomal breakage test will lead to an accurate diagnosis.

Dyserythropoiesis often representing immature erythroid components with megaloblastic change and/ or abnormal localization of erythropoiesis is observed.,24 Reduced megakaryocytes with dysplastic changes, such as micro-megakaryocytes, may be present which are easily identified by IHC staining of CD61 or CD42b. ${ }^{22}$ Reduced granulopoiesis is often observed and dysplastic changes are sometimes present. ${ }^{24}$ It is difficult to detect dysgranulopoiesis on bone marrow biopsy evaluation, but it has been reported that dysplasia consistently accompanies left-shifted granulopoiesis. ${ }^{4}$ considering these histological features it is not possible to reliably distinguish between FA and hypoplastic refractory cytopenia of childhood (RCC) by BM histology alone. The presence of mild dyshematopoiesis and lack of increase in blasts are overlapping features observed in both FA and RCC. ${ }^{24}$ In a study, FA was diagnosed in $14.5 \%$ of patients with histomorphological findings consistent with hypo/normocellular RCC. ${ }^{25}$ Importantly, some of these FA patients did not have any family history or physical abnormalities. Thus to differentiate these two entities a chromosomal breakage test in addition to the histomorphological evaluation is required.

FA-related MDS usually undergoes a hypoplastic or aplastic phase followed by progression to the emergence of abnormal cytogenetic clones. ${ }^{24}$ A study showed that the association between MDS and cytogenetic abnormalities differed according to the morphological findings. They found that $100 \%$ of patients with increased blasts or dysgranulopoiesis had cytogenetic abnormalities as compared to $83 \%$ of patients with dysmegakaryopoiesis and $33 \%$ of patients with dyserythropoiesis. They concluded that the reliable diagnostic criteria for FA-related MDS were increased blasts and dysgranulopoiesis, followed by dysmegakaryopoiesis. ${ }^{24} \mathrm{~A}$ specific pattern of chromosomal and genomic abnormalities is associated with FA associated progression to MDS.26 1q+, 3q+, 21q/RUNX1 (cryptic RUNX1 rearrangement), -7/7q, and 11q- are abnormalities found in MDS/AML. $3 q+,-7 / 7 q$, and $21 q /$ RUNX1 are observed only in the MDS/AML stage, whereas $1 \mathrm{q}+$ can be found in all stages, including non-MDS normocellular/ hypocellular BM of FA. ${ }^{24}$ FA with only $1 \mathrm{q}+$ abnormality may go on for years without their disease progression to 
MDS/ AML.

Studies have found p53-positive cells in the BM of FA were similar to that of FA-non-related MDS/AML but higher than in normal control or aplastic anemia. ${ }^{27}$ As the overexpression of $\mathrm{p} 53$ protein has been reported in the $\mathrm{BM}$ of MDS/AML. ${ }^{28}$ Hence a common mechanism between FA and FA-non-related MDS/AML responsible for the preleukemic /leukemic processes may exist. ${ }^{27}$ However, it is still unclear as to whether p53 immunostaining can differentiate FA from other BMF syndromes or can be useful as a predictor of disease progression.

\section{Dysketatosis Congenita}

Dyskeratosis congenita (DC) is characterized clinically by mucocutaneous abnormalities (i.e., abnormal skin pigmentation, nail dystrophy, and leukoplakia), a predisposition to malignancies and bone marrow failure. ${ }^{29}$ Before the age of 10 years mucocutaneous abnormalities appear. ${ }^{3}$ About $80 \%$ of the individuals show signs before the age of 30 and BMF is the chief cause of mortality. While only one hematopoietic lineage may be affected initially but with the progression of the disease pancytopenia develops. The bone marrow may be normocellular to aplastic, resembling idiopathic aplastic anemia. The diagnosis can be challenging if no physical anomalies of DC are present. At least one physical abnormality is seen in approximately $75 \%$ of individuals with DC. ${ }^{1}$ The physical abnormalities are usually age-dependent in these patients, thus, the absence of this feature does not eliminate DC from the list of differential diagnoses in young individuals. ${ }^{1}$

The median age at presentation is 14 years. ${ }^{4}$ Cytopenias can occur before the appearance of physical abnormalities. Usually, thrombocytopenia is the initial presentation30 occurring within the first decade of life. By the age of 40 years, the cumulative incidence of BMF is approximately $45 \% .^{7}$ The cumulative incidence of leukemia and MDS is up to $10 \%$ by 70 years of age and $20 \%$ by 50 years of age, respectively. ${ }^{7}$ As in FA the most common solid tumors are head and neck SCC, and other tumors include tumors of the gastrointestinal and anogenital regions. ${ }^{1}$ By the age of 65 , the cumulative incidence of solid tumors is approximately $20 \% .^{7}$ The median age of survival is 49 years. ${ }^{1}$ BMF, pulmonary diseases, and malignancies are the causes of mortality. ${ }^{29}$ Pulmonary fibrosis is a rare manifestation associated with DC.

Dysketatosis congenita (DC) results from inherited mutations in telomere maintenance genes. ${ }^{29} \mathrm{~A}$ wide spectrum of diseases are associated with defective telomere biology, collectively known as telomere diseases. ${ }^{4}$ Classic DC represents the prototype of these diseases. Forms of diseases other than classic DC have been recognized namely Hoyeraal-Hreidarsson syndrome, a severe form of
DC, affecting multisystem organs. ${ }^{29}$ It is characterized by microcephaly, cerebellar hypoplasia, intrauterine growth retardation, severe aplastic anemia, and immunodeficiency. Reversz syndrome, another severe form, causes bilateral exudative retinopathy in addition to features of classic DC. ${ }^{31}$

A higher than average risk of mortality is associated with stem cell transplantation in DC possibly due to the underlying systemic telomere defect. Inheritance of DC can be as X-linked, autosomal dominant, and autosomal recessive forms. De novo mutations are also frequent. About $70 \%$ of individuals with DC have mutations in one of the telomere maintenance genes (DKC1, TERC, TERT, TINF2, NHP2, NOP10, WRAP53 (protein TCAB1), CTC1, and RTEL1). The X-linked recessive form associated with the $\mathrm{DKC} 1$ gene encoding a core component of telomerase protein dyskerin is the most common form of inheritance. ${ }^{3}$ Telomeres of individuals with DC are markedly shorter due to abnormal telomere maintenance, usually below the 1 st percentile. ${ }^{32}$ Mutation in one of the telomere genes associated with DC phenotype may be seen in up to $5 \%$ of patients with severe idiopathic aplastic anemia (AA) according to various studies. ${ }^{33}$ Thus, differentiating AA from DC by regular screening of telomere length is important prior to initiation of therapy of AA patients. Of the methods used to detect telomere length, flow-FISH has been shown to be the most beneficial in screening patients with aplastic anemia. ${ }^{5}$ Other non-DC IBMF syndromes such as DBA, SDS, and FA also show short telomerase. However, the non-DC patients show telomere length clusters in the low normal range in contrast to DC patients where the telomere length is below the $1^{\text {st }}$ percentile found in normal controls. ${ }^{32}$

Depending on the biopsy timing and the stage of the disease, DC can exhibit varying degrees of cellularity as in FA. BM is usually hypocellular, but hypercellularity can be seen in the early stage of the disease. Gradually or progressively BM may become hypocellular as the severity of BMF increases. ${ }^{10}$ Ultimately it may become indistinguishable from AA. A shortened length of telomere correlates with the severity of cytopenia but the correlation between the degree of aplasia in the BM and telomere length remains unclear. ${ }^{32}$ DC patients often display dysplasia in any hematopoietic cell lineage in the $\mathrm{BM}^{29,30}$ Immature erythroid clusters with megaloblastic changes and abnormal localization of these cells may be found. Granulopoiesis varies from reduced or normal to increased with accompanying leftshifted maturation. Reduced megakaryopoiesis with micromegakaryocytes and abnormal localization of these cells are found. ${ }^{1,22}$ As in FA, reliably distinguishing between DC and RCC by morphology alone may not be possible. Morphological features shared with RCC are a lack of increased blasts and the presence of dysplastic changes. The measurement of the telomere length along with careful clinical examination is required to differentiate the two diseases. Dysplasia in the bone marrow alone does not 
necessarily indicate progression to DC-related MDS. For the diagnosis of secondary MDS morphological abnormalities including a degree of dysplasia, and an increase in the number of blast cells or BM cellularity compared with the baseline $\mathrm{BM}$ is required. ${ }^{34}$ The presence of chromosomal abnormalities associated with sporadic MDS/AML, such as monosomy 7, or multiple chromosomal abnormalities is an indication of the development of malignancy. ${ }^{17,30}$

Studies evaluating p53 IHC staining in DC are limited..$^{27}$ As opposed to other IBMF disorders, DC has a negative correlation between p53 expression and the values of Ki-67 or survivin, an inhibitor of apoptosis proteins. ${ }^{27}$ Though the reason for this is unknown, these findings imply that the p53-mediated pathway is activated in DC, whereas there is impairment of the compensatory expression of Ki-67 and survivin against apoptosis. Defects in the rescue of such vulnerable DC cells may be the reason for an increased incidence of BMF in DC.

\section{Shwachman-Diamond Syndrome}

Shwachman-Diamond syndrome (SDS) is a ribosomopathy characterized by neutropenia, associated pancreatic exocrine function insufficiency, metaphyseal dysostosis, BMF, and predisposition to MDS/AML. ${ }^{35-37}$ Biallelic mutations in the SBDS gene, which functions in ribosome biogenesis are present in most patients with SDS. ${ }^{38}$ SDS can also affect the skeletal, cardiac, neurocognitive, gastrointestinal, and immune systems. $55 \%$ of patients with SDS have physical abnormalities. 1 The common physical abnormalities in descending order are short stature, metaphyseal dysostosis, thoracic abnormalities, and delayed development. 1 The hallmark of SDS is exocrine pancreatic dysfunction with or without malabsorption. ${ }^{37}$ Over $90 \%$ of patients with SDS are diagnosed with pancreatic dysfunction. They usually present as steatorrhea within the first 6 months of life. ${ }^{36,37}$ Malabsorption can occur from birth to 11 years, the median age at presentation is 2 weeks. ${ }^{1}$ The decreased secretion of proteolytic enzymes leads to steatorrhea. ${ }^{37}$ Pancreatic biopsies reveal fatty replacement of acinar cells, an increase in the interstitial connective tissue, and dilated ductules. ${ }^{39}$ Age-related improvement in pancreatic function is demonstrable in approximately $50 \%$ of patients with SDS, though the reason remains unclear. ${ }^{40}$

Neutropenia is the most common hematologic abnormality and although seen in all patients, it can be either intermittent or persistent with variable severity. ${ }^{41}$ Neutropenia often develops slightly later than malabsorption, but it may be observed in the neonatal period. Patients have impaired neutrophil chemotaxis too. Anemia is present in two-thirds of the patients, and intermittent thrombocytopenia in a third of them. Unlike other BMF syndromes like DBA and CDA, the anemia in SDS is associated with an appropriate bone marrow response and reticulocytosis. A majority of patients present with elevated hemoglobin $F$ levels and macrocytosis. The reported cumulative incidence of BMF is $40 \%$ by the age of $50 .^{7}$ The respective cumulative incidence of MDS and AML is $65 \%$ by the age of 50 years and $5 \%$ by 20 of age. $^{7}$ SDS may be less prone to developing cancer, but cohorts have been too small for risk assessment. Few cases of solid tumors in SDS patients have been reported. ${ }^{42,43}$ The cumulative incidence of solid tumors in SDS was approximately reported as $40 \%$ by 45 years of age. ${ }^{7}$ Median age for survival is 41 years. ${ }^{7}$ The major causes of mortality are infection (sepsis or pneumonia), AML, and myocardial necrosis. ${ }^{1}$

SDS diagnosis is based on the clinical features, especially exocrine pancreatic dysfunction and hematological manifestations, and confirmation is by SBDS genetic testing. ${ }^{37}$ Though the clinical diagnosis is usually made during infancy it may be established in older children and adults. Half of the patients with SDS will present with classic neutropenia associated with diarrhea. ${ }^{44}$ Sensitive tests for pancreatic dysfunction are serum trypsinogen and pancreatic isoamylase levels adjusted for age. ${ }^{37}$ Fatty replacement of the pancreas on imaging studies and a low concentration of fecal pancreatic enzyme are indicative of pancreatic dysfunction and supportive of the diagnosis. Approximately $90 \%$ of SDS patients are diagnosed by the presence of biallelic mutations in the SBDS gene. ${ }^{38}$ In the remaining $10 \%$ of patients, mutations of genes other than SDSB have been implicated. Biallelic mutations in DNAJC21, the 60S ribosome assembly factor, were recently identified in a subset of SDS patients. ${ }^{45}$

The bone marrow findings are overall non-specific. Cellularity varies; some patients are hypocellular, while others show normocellular or hypercellular marrow. Hypercellularity may be due to the increase in the number of normal B cell progenitors, hematogones. ${ }^{5}$ The degree of cellularity is poorly correlated with the severity of peripheral cytopenia. ${ }^{46}$ Though BM hypocellularity in SDS is due to decreased granulopoiesis, a decrease in erythropoiesis and/or megakaryopoiesis is not uncommon. ${ }^{22,37}$ As seen in RCC, hypocellular BM in SDS often shows the patchy distribution of hematopoiesis. Dysgranulopoiesis presents as a shift to left with maturation arrest. Dyserythropoiesis is represented as megaloblastic changes or increased proerythroblasts and their abnormal localization. Similarly, dysmegakaryopoiesis shows micro-megakaryocytes and abnormal localization. Prominent multilineage dysplasia may indicate malignant transformation if seen in SDS. ${ }^{37}$ As with other IBMF syndromes like FA and DC, the distinction between SDS and RCC based on morphology alone is challenging. Patients diagnosed with RCC based on bone marrow examination may inaccurately include those with SDS.

SDS has an overall higher risk of developing bone marrow aplasia, myelodysplastic syndrome, and acute myeloid leukemia. Secondary AML presents as AML 
with MDS-related changes (AML-M2, AML-M4, AML-M6, AML-M0). ${ }^{47}$ AML-M6 frequently occurs in SDS patients. 47 The differentiation of MDS secondary to SDS may present a diagnostic dilemma as mild dysplastic changes are common and can be transient in SDS patients. Thus, their presence alone does not necessarily indicate secondary MDS. Further, not all cytogenetic abnormalities are indicative of malignant clonal transformation. SDS has been associated with cytogenetic abnormalities such as i(7) (q10) and $\operatorname{del}(20)(\mathrm{q} 11)$. These abnormalities may persist for years and their presence in isolation is not sufficient for subsequent development of MDS and AML. To establish the diagnosis of SDS-related MDS/AML, interpretation of cytogenetic abnormalities along with BM histomorphology should be made. ${ }^{37}$ Acquisition of well-known cytogenetic aberrations associated with MDS/AML, such as monosomy 7 , or multiple cytogenetic abnormalities along with the deterioration of histomorphological changes compared with the baseline BM, should be considered warning features of secondary MDS/AML..$^{17,47}$

In cases of SDS, BM biopsies were p53-positive on IHC. 48 In SDS, IHC expression levels of p53, Ki67, and survivin were positively correlated. ${ }^{27}$ These findings support the fact that survivin is elevated to overcome p53-dependent apoptosis and promote cell proliferation, which presents as elevated Ki67. The reduced incidence of BMF in SDS may be due to this retained function which compensates for p53-dependent apoptosis. Osteoporosis characterized by a reduced trabecular bone volume, low numbers of osteoclasts and osteoblasts, and a reduced amount of osteoid may be found in bone biopsies of patients with SDS. ${ }^{49}$ Osteoporosis may be associated with BM dysfunction and neutropenia or it may represent a primary defect in bone metabolism.

The differential diagnosis of SDS is other IBMF syndromes associated with failure of myelopoiesis and isolated neutropenia such as the severe congenital neutropenia syndromes (SCN). In patients with pancytopenia and bone marrow aplasia, Fanconi anemia and dyskeratosis congenita have to be excluded. ${ }^{5}$ Severe congenital neutropenia (SCN) is a genetically and phenotypically heterogeneous disorder presenting in early infancy with severe neutropenia and infections. The diagnosis of SCN is based on the persistent severe neutropenia usually associated with monocytosis and eosinophilia in the peripheral blood and normocellular bone marrow demonstrating granulocytic lineage maturation arrest at promyelocyte/myelocyte stage. ${ }^{50}$ It has to differentiate with other causes of neutropenia in newborns and infancy such as infections, drug-induced neutropenia, alloimmune neutropenia, metabolic disorders, and congenital immunodeficiency syndromes.

\section{CONCLUSIONS}

Evaluation of a suspected inherited bone marrow failure syndrome can present a challenge. Comprehensive approach and concurrent assessment of BM histology as well as the clinical features, physical anomalies, and laboratory/ genetic findings, and comparison with the baseline BM are necessary for an accurate diagnosis. Ancillary studies such as cytogenetics, chromosome breakage studies, flow cytometry, telomere length measurement, and others may be needed. Moreover, these IBMF syndromes are often heritable, affecting other family members, thus requiring insightful genetic counseling.

\section{Conflict of interest: None}

\section{REFERENCES}

1. Shimamura A, Alter BP. Pathophysiology and management of inherited bone marrow failure syndromes. Blood Rev. 2010; 24:101122. Crossref

2. Leguit RJ, van den Tweel JG. The pathology of bone marrow failure. Histopathology. 2010; 57:655-670. $\underline{\text { Crossref }}$

3. Iwafuchi H. The histopathology of bone marrow failure in childrenJ. clin. exp. hematop.2018; 58: 68-86. $\underline{\text { Crossref }}$

4. Cantu C, Proytcheva MA. Bone marrow failure syndromes, a practical approach to diagnosis J Hematopathol. 2015; 8:101-12. $\underline{\text { Crossref }}$

5. Vlachos A, Ball SE, Dahl N et al. Diagnosing and treating Diamond Blackfan anaemia: results of an international clinical consensus conference. Br J Haematol. 2008; 142:859-76. Crossref

6. Vlachos A, Rosenberg PS, Atsidaftos E, Alter BP, Lipton JM. Incidence of neoplasia in Diamond Blackfan anemia: a report from the Diamond Blackfan Anemia Registry. Blood. 2012; 119:3815-9. $\underline{\text { Crossref }}$

7. Alter BP, Giri N, Savage SA, Rosenberg PS. Cancer in the National Cancer Institute inherited bone marrow failure syndrome cohort after fifteen years of follow-up. Haematologica. 2018; 103:30-9. Crossref

8. Dong HY, Wilkes S, Yang H. CD71 is selectively and ubiquitously expressed at high levels in erythroid precursors of all maturation stages: a comparative immunochemical study with glycophorin A and hemoglobin A. Am J Surg Pathol. 2011; 35:723-32. Crossref

9. Rosse C, Kraemer MJ, Dillon TL, McFarland R, Smith NJ. Bone marrow cell populations of normal infants; the predominance of lymphocytes. J Lab Clin Med. 1977; 89:1225-40. Crossref

10. Foucar K, Viswanatha DS, Wilson CS. Non-Neoplastic Disorders of Bone Marrow (Atlas of Nontumor Pathology). 1st series. Washington, DC, American Registry of Pathology. 2008; 221-48.

11. Dutt $\mathrm{S}$, Narla $\mathrm{A}$, Lin $\mathrm{K}$, et al. Haploinsufficiency for ribosomal protein genes causes selective activation of $\mathrm{p} 53$ in human erythroid progenitor cells. Blood. 2011; 117:2567-2576. Crossref 
12. Van den Akker M, Dror Y, Odame I. Transient erythroblastopenia of childhood is an underdiagnosed and self-limiting disease. Acta Paediatr. 2014; 103:288-94. Crossref

13. Lipton JM, Ellis SR. Diamond-Blackfan anemia: diagnosis, treatment, and molecular pathogenesis. Hematol Oncol Clin North Am. 2009; 23:261-82. $\underline{\text { Crossref }}$

14. Shaw J, Meeder R. Transient erythroblastopenia of childhood in siblings: case report and review of the literature. J Pediatr Hematol Oncol. 2007; 29:659-60. $\underline{\text { Crossref }}$

15. Foot AB, Potter MN, Ropner JE, Wallington TB, Oakhill A. Transient erythroblastopenia of childhood with CD10, TdT, and cytoplasmic mu lymphocyte positivity in bone marrow. J Clin Pathol. 1990;43:857-9. Crossref

16. Dickerman JD. Transient erythroblastopenia of childhood presenting with reticulocytosis and erythroid hyperplasia in the bone marrow. Pediatrics. 1981;67:562-4. Website

17. Godley LA, Shimamura A. Genetic predisposition to hematologic malignancies: management and surveillance. Blood. 2017; 130:424432. Crossref

18. Babushok DV, Bessler M, Olson TS. Genetic predisposition to myelodysplastic syndrome and acute myeloid leukemia in children and young adults. Leuk Lymphoma. 2016; 57:520-36. Crossref

19. Chirnomas S, Kupfer G. The inherited bone marrow failure syndromes. Pediatr Clin North Am. 2013; 60:1291-310. Crossref

20. Soulier J. Fanconi anemia. Hematology Am Soc Hematol Educ Program. 2011; 2011:492-497. $\underline{\text { Crossref }}$

21. Kutler DI, Singh B, Satagopan J, et al. A 20-year perspective on the International Fanconi Anemia Registry (IFAR). Blood. 2003; 101:124912-56. $\underline{\text { Crossref }}$

22. Hashmi SK, Allen C, Klaassen R, et al. Comparative analysis of Shwachman-Diamond syndrome to other inherited bone marrow failure syndromes and genotype-phenotype correlation. Clin Genet. 2011; 79:448-58. Crossref

23. Butturini A, Gale RP, Verlander PC, et al. Hematologic abnormalities in Fanconi anemia: an International Fanconi Anemia Registry study. Blood. 1994; 84:1650-5. Website

24. Cioc AM, Wagner JE, MacMillan ML, DeFor T, Hirsch B. Diagnosis of myelodysplastic syndrome among a cohort of 119 patients with fanconi anemia: morphologic and cytogenetic characteristics. Am J Clin Pathol. 2010; 133:92-100. Crossref

25. Yoshimi A, Niemeyer C, Baumann I, et al. High incidence of Fanconi anaemia in patients with a morphological picture consistent with refractory cytopenia of childhood. Br J Haematol. 2013; 160:109-11. Crossref
26. Quentin S, Cuccuini W, Ceccaldi R, et al. Myelodysplasia and leukemia of Fanconi anemia are associated with a specific pattern of genomic abnormalities that includes cryptic RUNX1/ AML1 lesions. Blood. 2011; 117:161-70. Crossref

27. Al-Rahawan MM, Alter BP, Bryant BJ, Elghetany MT. Bone marrow cell cycle markers in inherited bone marrow failure syndromes. Leuk Res. 2008; 32:1793-9. $\underline{\text { Crossref }}$

28. Kurotaki H, Tsushima Y, Nagai K, Yagihashi S. Apoptosis, bcl-2 expression and p53 accumulation in myelodysplastic syndrome, myelodysplastic-syndrome-derived acute myelogenous leukemia and de novo acute myelogenous leukemia. Acta Haematol. 2000; 102:115-23. Crossref

29. Dokal I. Dyskeratosis congenita. Hematology Am Soc Hematol Educ Program. 2011; 2011:480-6. Crossref

30. Savage SA, Cook EF (eds). Dyskeratosis congenital and telomere biology disorders: Diagnosis and management guidelines. Website

31. Revesz T, Fletcher S, al-Gazali LI, DeBuse P. Bilateral retinopathy, aplastic anaemia, and central nervous system abnormalities: a new syndrome? J Med Genet. 1992; 29:673-5. Crossref

32. Alter BP, Giri N, Savage SA, Rosenberg PS. Telomere length in inherited bone marrow failure syndromes. Haematologica 2015; 100:49-54. $\underline{\text { Crossref }}$

33. Sakaguchi H, Nakanishi K, Kojima S. Inherited bone marrow failure syndromes in 2012. Int J Hematol. 2013; 97:20-9. Website

34. Erlacher M, Strahm B. Missing Cells: Pathophysiology, Diagnosis, and Management of (Pan)Cytopenia in Childhood. Front Pediatr. 2015;3:64. Crossref

35. Burwick N, Shimamura A, Liu JM. Non-Diamond Blackfan anemia disorders of ribosome function: Shwachman Diamond syndrome and 5q- syndrome. Semin Hematol. 2011; 48:136-43. Crossref

36. Myers KC, Davies SM, Shimamura A. Clinical and molecular pathophysiology of Shwachman-Diamond syndrome: an update. Hematol Oncol Clin North Am. 2013; 27:117-28. Crossref

37. Dror Y, Donadieu J, Koglmeier J, et al. Draft consensus guidelines for diagnosis and treatment of Shwachman-Diamond syndrome. Ann N Y Acad Sci. 2011; 1242:40-55. $\underline{\text { Crossref }}$

38. Boocock GR, Morrison JA, Popovic M, et al. Mutations in SBDS are associated with Shwachman-Diamond syndrome. Nat Genet. 2003; 33:97-101. Crossref

39. Aggett PJ, Cavanagh NP, Matthew DJ, et al. Shwachman's syndrome. A review of 21 cases. Arch Dis Child. 1980; 55:331-47. Website

40. Mack DR, Forstner GG, Wilschanski M, Freedman MH, Durie PR. Shwachman syndrome: exocrine pancreatic dysfunction and variable 
phenotypic expression. Gastroenterology. 1996; 111:1593-602. Crossref

41. Smith OP, Hann IM, Chessells JM, Reeves BR, Milla P. Haematological abnormalities in Shwachman-Diamond syndrome. Br J Haematol. 1996; 94:279-84. Crossref

42. Singh SA, Vlachos A, Morgenstern NJ, et al. Breast cancer in a case of Shwachman Diamond syndrome. Pediatr Blood Cancer. 2012; 59:945-6. Crossref

43. Nakaya T, Kurata A, Hashimoto $H$, et al. Young-age-onset pancreatoduodenal carcinoma in Shwachman-Diamond syndrome. Pathol Int. 2014; 64:75-80. DOI: 10.1111/pin.12133

44. Myers KC, Bolyard AA, Otto B, et al. Variable clinical presentation of Shwachman-Diamond syndrome: update from the North American Shwachman-Diamond Syndrome Registry. J Pediatr. 2014; 164:86670. Crossref

45. Dhanraj S, Matveev A, Li H, et al. Biallelic mutations in DNAJC21 cause Shwachman-Diamond syndrome. Blood. 2017; 129:1557-62. $\underline{\text { Crossref }}$
46. Dror Y, Freedman MH. Shwachman-Diamond syndrome: An inherited preleukemic bone marrow failure disorder with aberrant hematopoietic progenitors and faulty marrow microenvironment. Blood. 1999; 94:3048-54. Website

47. Donadieu J, Fenneteau O, Beaupain B, et al. Classification of and risk factors for hematologic complications in a French national cohort of 102 patients with Shwachman-Diamond syndrome. Haematologica. 2012; 97:1312-9. Crossref

48. Elghetany MT, Alter BP. p53 protein overexpression in bone marrow biopsies of patients with Shwachman-Diamond syndrome has a prevalence similar to that of patients with refractory anemia. Arch Pathol Lab Med. 2002; 126:452-5. Crossref

49. Toiviainen-Salo S, Mäyränpää MK, Durie PR, et al. ShwachmanDiamond syndrome is associated with low-turnover osteoporosis. Bone. 2007; 41:965-72. Crossref

50. Welte K, Zeidler C. Severe congenital neutropenia. Hematol Oncol Clin North 2009; 23:307-20. Crossref 